\title{
Multiscale resolution in the computation of crystalline microstructure
}

\section{Journal Article}

Author(s):

Bartels, Sören; Prohl, Andreas

Publication date:

2004

Permanent link:

https://doi.org/10.3929/ethz-b-000422916

Rights / license:

In Copyright - Non-Commercial Use Permitted

Originally published in:

Numerische Mathematik 96(4), https://doi.org/10.1007/s00211-003-0483-8 


\title{
Multiscale resolution in the computation of crystalline microstructure
}

\author{
Sören Bartels ${ }^{1}$, Andreas Prohl $^{2}$ \\ 1 Mathematisches Seminar, Christian-Albrechts-Universität zu Kiel, \\ Ludewig-Meyn-Str. 4, 24098 Kiel, Germany; e-mail: sba@ numerik.uni-kiel.de \\ 2 Department of Mathematics, ETHZ, 8092 Zurich, Switzerland; \\ e-mail: apr@math.ethz.ch
}

Received February 12, 2002 / Revised version received February 14, 2003 / Published online August 5, 2003 - (c) Springer-Verlag 2003

Summary. This paper addresses the numerical approximation of microstructures in crystalline phase transitions without surface energy. It is shown that branching of different variants near interfaces of twinned martensite and austenite phases leads to reduced energies in finite element approximations. Such behavior of minimizing deformations is understood for an extended model that involves surface energies. Moreover, the closely related question of the role of different growth conditions of the employed bulk energy is discussed. By explicit construction of discrete deformations in lowest order finite element spaces we prove upper bounds for the energy and thereby clarify the question of the dependence of the convergence rate upon growth conditions and lamination orders. For first order laminates the estimates are optimal.

Mathematics Subject Classification (2000): 65K10, 65M50, 65N30, 73C50, $73 \mathrm{~S} 10$

\section{Introduction}

The mathematical model of phase transitions in crystalline solids leads to the minimization of a non-quasiconvex functional over a space of admissible deformations. Following the work of $[1,2]$ we consider the variational problem: Find $u \in \mathcal{A}$ such that $I(u)=\inf _{v \in \mathcal{A}} I(v)$. Here,

$$
\mathcal{A}:=\left\{v \in W^{1, p}\left(\Omega ; \mathbb{R}^{n}\right): v(x)=F x \text { for all } x \in \partial \Omega\right\},
$$

Correspondence to: A. Prohl 
where $\Omega \subseteq \mathbb{R}^{n}, n=2,3$, is a bounded Lipschitz domain, $F \in \mathbb{R}^{n \times n}$ is a given homogeneous deformation gradient and serves as the boundary data. For $v \in W^{1, p}\left(\Omega ; \mathbb{R}^{n}\right)$ the energy functional $I: W^{1, p}\left(\Omega ; \mathbb{R}^{n}\right) \rightarrow \mathbb{R}$ is defined by

$$
I(v):=\int_{\Omega} W_{\vartheta}(\nabla v) d x .
$$

$W_{\vartheta}$ is a temperature-dependent, continuous energy density such that, for all $G \in \mathbb{R}^{n \times n}$, there holds

$$
c_{1}|G|^{p}-c_{2} \leq W_{\vartheta}(G) \leq c_{2}\left(|G|^{p}+1\right)
$$

with constants $c_{1}, c_{2}>0$ and $p \geq 1$. Different choices of $W_{\vartheta}$ model various phase transitions in crystalline solids. We refer to [19] for the Ericksen-James energy density that describes a cubic to tetragonal phase transition and restrict ourselves to $n=2$ and temperature independent energy densities of the form

$$
W_{\vartheta}(G)=W(G)=\operatorname{dist}\left(G,\left\{F_{1}, \ldots, F_{N}\right\}\right)^{p}, \quad G \in \mathbb{R}^{2 \times 2},
$$

with a positive integer $N$ and given (compatible if $N=2$ ) wells $F_{1}, \ldots, F_{N} \in$ $\mathbb{R}^{2 \times 2}$. Note that this definition of $W_{\vartheta}$ is note frame indifferent but appears as a good model example for non-convex energy densities.

It is due to non-quasiconvexity of $W$ that the variational problem does in general not admit solutions and infimizing sequences reveal the most important information of the physical situation. A conforming finite element scheme replaces $\mathcal{A}$ by a finite-dimensional subspace $\mathcal{A}_{h}$ and the resulting minimization problem admits a solution. For a family of finite element spaces $\left(\mathcal{A}_{h}\right)_{h>0}$ there holds $\lim _{h \rightarrow 0} \inf _{v_{h} \in \mathcal{A}_{h}} I\left(v_{h}\right) \rightarrow \inf _{v \in \mathcal{A}} I(v)$, i.e., finite element schemes yield infimizing sequences. Our main result concerns the decay rate of the energies and its dependence on the number $N \geq 2$ of wells and the exponent $p \geq 1$. We assume throughout this work that $F$ lies in the lamination convex hull $\left\{F_{j}: j=1, \ldots, N\right\}^{l c}$ (cf. [21] and Section 4 for a definition of $\left.K^{l c}\right)$ of $\left\{F_{j}: j=1, \ldots, N\right\}$ so that $\inf _{v \in \mathcal{A}} I(v)=0$.

The a priori analysis in [19] for $N=2$ states

$$
\inf _{v_{h} \in \mathcal{A}_{h}} I\left(v_{h}\right) \leq C h^{1 / 2},
$$

independently of $p$. Here, $h$ is the maximal meshsize of the underlying triangulation and throughout this paper $C$ is a generic, $h$-independent constant. It is shown in [10] that this estimate is sharp for $p=1$ in the sense that there exists a triangulation of the domain $\Omega:=(0,1)^{2} \subseteq \mathbb{R}^{2}$ and matrices $F_{1}, F_{2} \in \mathbb{R}^{2 \times 2}$ such that $\inf _{v_{h} \in \mathcal{A}_{h}} I\left(v_{h}\right) \geq C h^{1 / 2}$. In case that $N \geq 2$, the analysis in [16] shows

$$
\inf _{v_{h} \in \mathcal{A}_{h}} I\left(v_{h}\right) \leq C h^{1 /(1+L)}
$$


where $L \geq 1$ is the lamination level which is related to $F$ and $F_{1}, \ldots, F_{N}$ (cf. Section 4). In [8] the numerical analysis of a variational problem with incompatible wells $F_{1}, \ldots, F_{4}$ and $F \notin\left\{F_{1}, \ldots, F_{4}\right\}^{l c}$ leads to an upper bound $e^{-C|\ln h|^{1 / 2}}$. In all cited works, finite element minimizers are constructed (iteratively) by a lamination process and appropriate cut-off functions to satisfy the (averaged) boundary condition.

In physical experiments one observes branching of different variants near interfaces [24], and this effect has been analyzed in the continuous case in $[13,14,4]$ for energy densities that involve a surface energy term. We show that finite element deformations which exhibit similar branching structures on mesh dependent scales give rise to significantly reduced energies. In particular, we find an explicit dependence of branching structures on growth conditions.

Theorem 1.1 If $F \in\left\{F_{1}, \ldots, F_{N}\right\}^{l c}$ with lamination level $L$, and if $h$ is sufficiently small we have

$$
\inf _{v_{h} \in \mathcal{A}_{h}} I\left(v_{h}\right) \leq C h^{p /(p+L)} .
$$

It is conjectured that a similar estimate holds for $n=3$. We draw the following conclusions from our analysis: (i) We observe that the scale induced by the finite element space may be regarded as a scale arising from a surface energy. (ii) Finite element minimizers exhibit multiscale phenomena with branching structures close to the boundary and interfaces. (iii) The result is in agreement with the widely accepted conjecture that growth conditions are related to the amount of energy stored in interfaces between different phases and that they affect the geometry of branchings near interfaces. In particular, branching structures of constructed deformations disappear for the case $p=1$ which fits with the known constructions mentioned above.

In the case of two compatible energy wells our construction may be summarized as follows. We choose a coarse lamination in the interior of the domain of scale $\mathcal{O}\left(h^{1 /(p+1)}\right)$. In a boundary layer of thickness $\mathcal{O}\left(h^{p /(p+1)}\right)$ we choose a fine lamination of scale $\mathcal{O}\left(h^{p /(p+1)}\right)$. To interpolate between the coarse and the fine lamination we introduce a branching or refinement region of thickness $\mathcal{O}(1)$. In this refinement region the deformation gradient is not an element of $\left\{F_{1}, F_{2}\right\}$ but is close enough to the wells. The growth condition for the energy density enters the estimate through the distance of the deformation gradient to the wells in this region and thus determines the geometry of the branching. We then employ a sharp cut-off function in the boundary layer to satisfy the boundary conditions. Finally, we use a nodal interpolation operator and prove the estimate (1.1) for the resulting discrete deformation.

The techniques of [10] to prove inverse estimates for $p=L=1$ may be generalised to the case $p>1$ (see [3]) and show that our estimate is sharp 
for simple laminates in the sense of the following theorem. The idea of the proof is to use a special criss-cross triangulation and then to count the number of triangles on which the discrete deformation gradient does not equal one of the two wells. This number is estimated by changes of the gradient from one well to the other along lines that are orthogonal to the direction in which oscillations occur.

Theorem 1.2 There exist $F_{1}, F_{2} \in \mathbb{R}^{2 \times 2}, F=\left(F_{1}+F_{2}\right) / 2$, and a triangulation $\mathcal{T}$ of $\Omega=(0,1)^{2}$ such that, for each $v_{h} \in \mathcal{A}_{h}$ satisfying

$$
I\left(v_{h}\right) \leq C h^{p /(p+1)},
$$

there holds

$$
I\left(v_{h}\right) \geq C^{\prime} h^{p /(p+1)} .
$$

Proof. We refer to [10] for a proof if $p=1$. It has been verified in [3] that the arguments of [10] also prove the theorem if $p>1$.

We stress that our analysis is of theoretical interest: It is unlikely that a numerical scheme will find a correct minimizer. Using a priori knowledge, branching has been observed in numerical experiments in [18]. For the efficient computation of generalized formulations and their relation to the original problem we refer to $[3,5,6,15]$.

The rest of the paper is organized as follows: In Section 2 we introduce some notation and definitions and prove a basic lemma that shows our energy estimate (1.1) for simple laminates $(N=2)$ and allows for iteration $(N>2)$. The application of this lemma to a three well problem is performed in Section 3 to illustrate one iteration step. In Section 4 we show estimate (1.1) as the main result of this work.

\section{Basic lemma}

In this and in the following two sections we assume that $\Omega \subseteq \mathbb{R}^{2}$ is a convex, piecewise affine, bounded Lipschitz domain. Moreover, we assume that $\mathcal{T}$ is a regular triangulation of $\Omega$.

Definition 2.1 For $\omega \subseteq \mathbb{R}^{n}$ and $n, n^{\perp} \in \mathbb{R}^{n},|n|=\left|n^{\perp}\right|=1, n \cdot n^{\perp}=0$, the diameter of $\omega$ in direction $n$ is defined by the minimal distance of two half-spaces, given by points $z_{1}, z_{2}$ and the normal $n$, that exclude $\omega$, i.e.,

$$
\begin{gathered}
d_{n}(\omega):=\inf \left\{\operatorname{dist}\left(H_{1}, H_{2}\right): H_{j}=\left\{x \in \mathbb{R}^{n}:(-1)^{j}\left(x-z_{j}\right) \cdot n \leq 0\right\},\right. \\
\left.H_{j} \cap \omega=\emptyset, z_{j} \in \mathbb{R}^{n}, j=1,2\right\} .
\end{gathered}
$$

The maximal length of a line segment in $\omega$ parallel to $n^{\perp}$ is defined by

$$
\ell_{n^{\perp}}(\omega):=\sup \left\{|s|: s \in \mathbb{R}, x \in \omega, x+s n^{\perp} \in \omega\right\} .
$$


Remark 2.1 The quantity $s^{-1} d_{n}(\omega)$ describes how many line segments parallel to $n^{\perp}$ and in distances $s>0$ can be arranged in $\omega$ while $\ell_{n^{\perp}}(\omega)$ is the maximal length of such line segments (cf. Figure 1).

Lemma 2.1 (Basic Lemma) Let $\omega \subseteq \mathbb{R}^{2}$ be a convex bounded Lipschitz domain with piecewise affine boundary. Moreover, let $\tau$ be a regular triangulation that covers $\omega$ and let $h$ be the maximal diameter of elements in $\tau$. Let $F=\lambda F_{1}+(1-\lambda) F_{2}$ for $F_{1}, F_{2} \in \mathbb{R}^{2 \times 2}$ satisfying $F_{1}-F_{2}=a \otimes n$ for $a, n \in \mathbb{R}^{2},|n|=1$, and $\lambda \in(0,1)$. Assume that $n^{\perp} \in \mathbb{R}^{2}$ satisfies $\left|n^{\perp}\right|=1$ and $n \cdot n^{\perp}=0$. If $h$ is small enough there exists $y_{h} \in \mathcal{S}^{1}(\tau)^{2}$ with $y_{h}(x)=F x$ for all $x \in \partial \omega$ and such that for $\alpha \in[0,1], \gamma \in[0, \alpha)$, and $\delta \in[\alpha, 1]$ with $1>\frac{\delta-\alpha}{\delta-\gamma} \geq \frac{p-1}{p},(\alpha-\delta) /(p-1)+\alpha-\gamma \geq 0$ if $p>1$ and $\alpha=\delta$ if $p=1$, and $h^{\alpha}, h^{\gamma}, h^{\delta} \leq \operatorname{diam}(\omega)$, there holds

$$
\begin{aligned}
& \int_{\omega} \operatorname{dist}\left(\nabla y_{h}(x),\left\{F_{1}, F_{2}\right\}\right)^{p} d x \\
& \quad \leq C\left(|\partial \omega| h^{\delta}+d_{n}(\omega)\left(h^{p \alpha-(p-1) \gamma}+h^{1+\gamma-\alpha}\right)+d_{n}(\omega) \ell_{n^{\perp}}(\omega) h^{1-\alpha}\right) .
\end{aligned}
$$

Remark 2.2 The three terms on the right hand side of the estimate of the lemma reflect energy contributions that arise from a boundary layer, branching structures, and internal layer interfaces, respectively. Notice that only the second contribution shows dependence on the growth parameter $p \geq 1$.

As a direct consequence we obtain an improved energy estimate for simple laminates.

Theorem 2.1 Under the assumptions ofLemma 2.1 (with $\omega=\Omega$ and $\tau=\mathcal{T})$ there holds

$$
\int_{\Omega} \operatorname{dist}\left(\nabla y_{h}(x),\left\{F_{1}, F_{2}\right\}\right)^{p} d x \leq C h^{p /(p+1)} .
$$

Proof. Choosing $\alpha=1 /(p+1), \delta=p /(p+1)$, and $\gamma=0$, the assertion follows from Lemma 2.1 .

Proof of Lemma 2.1. Let $\alpha \in[0,1], \gamma \in[0, \alpha)$, and $\delta \in[\alpha, 1]$. For $k \in \mathbb{Z}$ define

$$
\begin{aligned}
& \omega_{k}^{1}:=\left\{x \in \omega: k h^{\alpha}<x \cdot n \leq(k+\lambda) h^{\alpha}\right\}, \\
& \omega_{k}^{2}:=\left\{x \in \omega:(k+\lambda) h^{\alpha}<x \cdot n \leq(k+1) h^{\alpha}\right\},
\end{aligned}
$$

and $\omega_{k}:=\omega_{k}^{1} \cup \omega_{k}^{2}$ (cf. Figure 1).

Step 1: Construction of laminates in the interior of $\omega$. For $x \in \omega$, we define a deformation $\tilde{y}_{0}$, with $\chi_{I}$ being the 1-periodic extension of the characteristic function of $I \subseteq(0,1)$ on $(0,1)$ to $\mathbb{R}$, by

$$
\tilde{y}_{0}(x):=F_{1} x-a \int_{0}^{x \cdot n} \chi_{(\lambda, 1)}\left(t / h^{\alpha}\right) d t .
$$




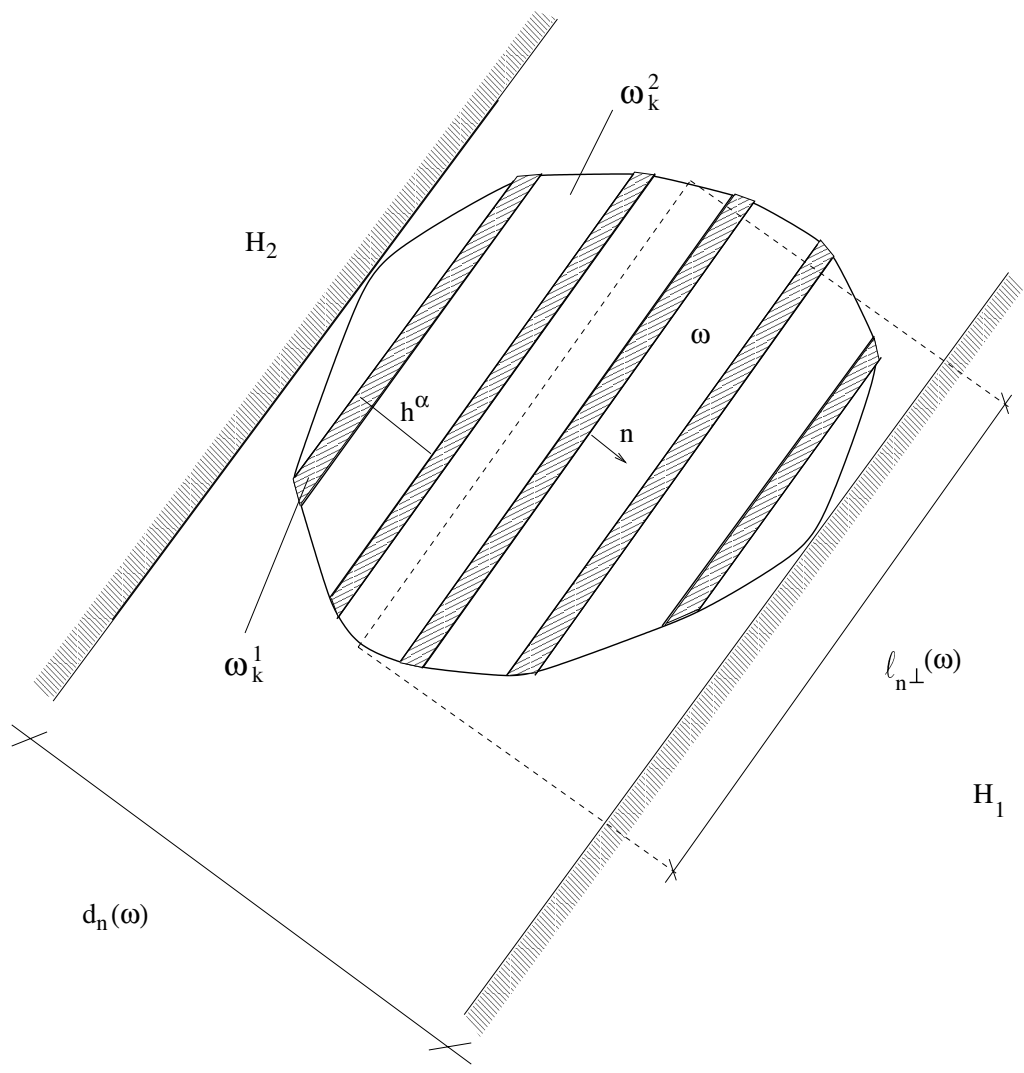

Fig. 1. Decomposition of $\omega$ and quantities $d_{n}(\omega), \ell_{n^{\perp}}(\omega)$

The mapping $\tilde{y}_{0}$ has the following properties.

(i) There holds $\tilde{y}_{0} \in W^{1, \infty}(\omega)$.

(ii) There holds $\nabla \tilde{y}_{0}=F_{1}-\chi_{(\lambda, 1)}\left(\frac{x \cdot n}{h^{\alpha}}\right) a \otimes n=F_{1}$ in $\omega_{k}^{1}$ and $\nabla \tilde{y}_{0}=F_{2}$ in $\omega_{k}^{2}$ for each $k \in \mathbb{Z}$ with $\omega_{k}^{1} \neq \emptyset$ respectively $\omega_{k}^{2} \neq \emptyset$. Thereby, $\omega$ is divided into at most $2 d_{n}(\omega) h^{-\alpha}$ convex subdomains $\omega_{k}^{j}, j=1,2$, in which $\nabla \tilde{y}_{0}$ is constant. The separating interfaces have a maximal length $\ell_{n^{\perp}}(\omega)$.

(iii) For all $m, m^{\perp} \in \mathbb{R}^{2},|m|=\left|m^{\perp}\right|=1, m \cdot m^{\perp}=0$, with $m \bigvee n n$ there holds $\ell_{m^{\perp}}\left(\omega_{k}^{j}\right) \leq C h^{\alpha}$ (where $C$ depends on $m$ and $n$ ) and $d_{m}\left(\omega_{k}^{j}\right) \leq d_{m}(\omega)$, $j=1,2$ and $k$ such that $\omega_{k}^{j} \neq \emptyset$.

The properties (i) and (ii) follow directly from the definition of $\tilde{y}_{0}$. Since each domain $\omega_{k}^{j}$ lies between two hyperplanes which are orthogonal to $n^{\perp}$ and which have a distance $h^{\alpha}$ from each other and since $m^{\perp} \not \mid n^{\perp}$ we have $\ell_{m^{\perp}}\left(\omega^{\ell}\right) \leq C h^{\alpha}$, i.e., we have (iii). 
We will first modify $\tilde{y}_{0}$ in a neighbourhood of $\partial \omega$ and then apply a sharp cut-off function so that it satisfies the boundary condition. Finally, we prove the asserted estimate for $y_{h}:=\mathcal{I}_{h} y$, the nodal interpolant of $y$.

Step 2: Construction of branchings for "twinned martensite - austenite" interfaces. For $A \subseteq \mathbb{R}^{2}$ let

$$
d_{n^{\perp}}(x, A):=\inf _{t \in \mathbb{R}: x+t n^{\perp} \in A}|t|
$$

denote the distance of $x \in \mathbb{R}^{2}$ to $A \subseteq \mathbb{R}^{2}$ in the direction of $n^{\perp}$ if it exists and $d_{n^{\perp}}(x, A):=+\infty$ otherwise. Let $t$ be the unit tangent to $\partial \omega$. For each connected component $\Gamma_{r, k}, r=1,2$, of $\partial \omega \cap \partial \omega_{k}$ we define a function $\tilde{y}_{r, k}$ in a neighbourhood of $\Gamma_{r, k}$ as follows.

(a) Assume that $\Gamma_{r, k}$ is affine, $\left.t\right|_{\Gamma_{r, k}} \backslash X n^{\perp}$, and $\ell_{n^{\perp}}\left(\omega_{k}\right) \geq 5 h^{\gamma}$. The domain

$$
B_{r, k}:=\omega_{k} \cap\left\{x \in \omega: d_{n^{\perp}}\left(x, \Gamma_{r, k}\right) \leq h^{\delta}+2 h^{\gamma}\right\},
$$

is assumed to be simply connected (i.e., $h$ is assumed to be small enough) and we decompose it as follows. Fix $s_{1} \in((p-1) / p, 1)$ and set $\theta:=2^{1 / s_{1}}$. Then, let

$$
\omega_{r, k}^{K+1}:=\left\{x \in B_{r, k}: d_{n^{\perp}}\left(x, \Gamma_{r, k}\right) \leq h^{\delta}\right\} .
$$

Here, $K \in \mathbb{N}, \bar{\varepsilon} \in[0,1)$ satisfy $K=\log _{2}\left(h^{\alpha-\delta)}\right)+\bar{\varepsilon}$. For $j=0, \ldots, K$ set

$$
\begin{aligned}
& \omega_{r, k}^{j}:=\left\{x \in B_{r, k}: \sum_{\ell=1}^{K-j} \frac{h^{\gamma}}{\theta^{K-\ell+1}}<d_{n^{\perp}}\left(x, \Gamma_{r, k}\right)-h^{\delta} \leq \sum_{\ell=1}^{K-j+1} \frac{h^{\gamma}}{\theta^{K-\ell+1}}\right\} \\
& \omega_{r, k}^{-1}:=\left\{x \in B_{r, k}: h^{\delta}+\sum_{\ell=0}^{K} \frac{h^{\gamma}}{\theta^{\ell}}<d_{n^{\perp}}\left(x, \Gamma_{r, k}\right)\right\}
\end{aligned}
$$

(cf. Figure 3). For $0 \leq s \leq 1$ the intervals $I_{1}(s)$ and $I_{2}(s)$ are defined by (cf. Figure 2)

$$
\begin{aligned}
& I_{1}(s):=s \frac{\lambda}{4}+(1-s) \frac{\lambda}{2}+\left(0, \frac{1-\lambda}{2}\right), \\
& I_{2}(s):=s\left(1-\frac{\lambda}{4}\right)+(1-s)\left(1-\frac{\lambda}{2}\right)-\left(\frac{1-\lambda}{2}, 0\right) .
\end{aligned}
$$

An important fact for the subsequent construction of branching laminates is that $I_{1}(1)=\frac{1}{2}\left(I_{1}(0) \cup I_{2}(0)\right)$. With the help of $I_{1}$ and $I_{2}$ the mapping $\tilde{y}_{r, k}: B_{r, k} \rightarrow \mathbb{R}^{2}$ is, with

$$
d_{j}(x)=\frac{\theta^{j}}{h^{\gamma}} d_{n^{\perp}}\left(x, \partial \omega_{r, k}^{j} \cap \partial \omega_{r, k}^{j-1}\right)
$$




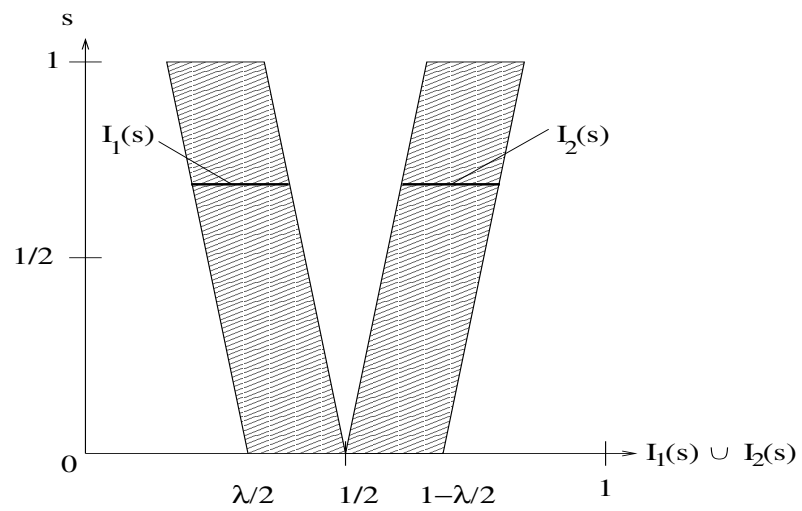

Fig. 2. Intervals $I_{1}(s)$ and $I_{2}(s)$ for the definition of branchings near the boundary

for values $0 \leq j \leq K$, defined as follows,

$$
\tilde{y}_{r, k}(x):=F_{1} x- \begin{cases}a \int_{0}^{x \cdot n} \chi_{(\lambda, 1)}\left(\frac{s}{h^{\alpha}}\right) d s, & x \in \omega_{r, k}^{-1}, \\ a \int_{0}^{x \cdot n} \chi_{I_{1}\left(d_{j}(x)\right) \cup I_{2}\left(d_{j}(x)\right)}\left(\frac{2^{j} s}{2 h^{\alpha}}\right) d s, & x \in \omega_{r, k}^{j}, \\ a \int_{0}^{x \cdot n} \chi_{I_{1}(1) \cup I_{2}(1)}\left(\frac{2^{K} s}{2 h^{\alpha}}\right) d s, & x \in \omega_{r, k}^{K+1} .\end{cases}
$$

Figure 3 illustrates, how the function $\tilde{y}_{r, k}$ is constructed with the help of $I_{1}, I_{2}$ and with scaling and periodification to obtain a self-similar pattern for $\tilde{y}_{r, k}$ (cf. the construction in [13]).

(a1) There holds $\tilde{y}_{r, k} \in W^{1, \infty}\left(B_{r, k}\right)$.

(a2) For all $x \in \omega_{r, k}^{j}, j=0, \ldots, K$, we have

$$
\begin{aligned}
\nabla \tilde{y}_{r, k}(x)= & F_{1}-\chi_{I_{1}\left(d_{j}(x)\right) \cup I_{2}\left(d_{j}(x)\right)}\left(2^{j} x \cdot n /\left(2 h^{\alpha}\right)\right) a \otimes n \\
& +\frac{\lambda \theta^{j}}{2^{j+1}} h^{\alpha-\gamma}\left(\chi_{I_{2}\left(d_{j}(x)\right)}\left(2^{j} x \cdot n /\left(2 h^{\alpha}\right)\right)\right. \\
& \left.+\chi_{I_{1}\left(d_{j}(x)\right)}\left(2^{j} x \cdot n /\left(2 h^{\alpha}\right)\right)\right) a \otimes n^{\perp},
\end{aligned}
$$

and $(\theta / 2)^{j} h^{\alpha-\gamma} \leq C, j=0, \ldots, K$. The mapping $\tilde{y}_{r, k}$ divides $\omega_{r, k}^{j}$ into at most $3 \cdot 2^{j}$ convex domains $\omega_{r, k}^{j, \ell}, \ell=1, \ldots, L_{j} \leq 3 \cdot 2^{j}$, of measure $\sim h^{\alpha} h^{\gamma} / 2^{j} \theta^{j}$ in which $\nabla \tilde{y}_{r, k}$ is constant. The joint boundaries have a maximal length $C h^{\gamma} / \theta^{j}$.

(a3) For all $m, m^{\perp} \in \mathbb{R}^{2},|m|=\left|m^{\perp}\right|=1, m \cdot m^{\perp}=0$, with $m \not \mid n$ there holds $\ell_{m^{\perp}}\left(\omega_{r, k}^{j, \ell}\right) \leq C h^{\alpha} / 2^{j}$ and $d_{m}\left(\omega_{r, k}^{j, \ell}\right) \leq C h^{\gamma} / \theta^{j}, j=1, \ldots, K$ and $\ell=1, \ldots, L_{j}$.

(a4) There holds $\left|\tilde{y}_{r, k}(x)-F x\right| \leq C h^{\alpha} / 2^{K} \leq C h^{\delta}$ for all $x \in \omega_{r, k}^{K+1}$.

(a5) There holds $\left.\tilde{y}_{r, k}\right|_{\partial B_{r, k} \cap \omega}=\left.\tilde{y}_{0}\right|_{\partial B_{r, k} \cap \omega}$ and $\tilde{y}_{0}=\tilde{y}_{r, k}$ in $\omega_{r, k}^{-1}$. 


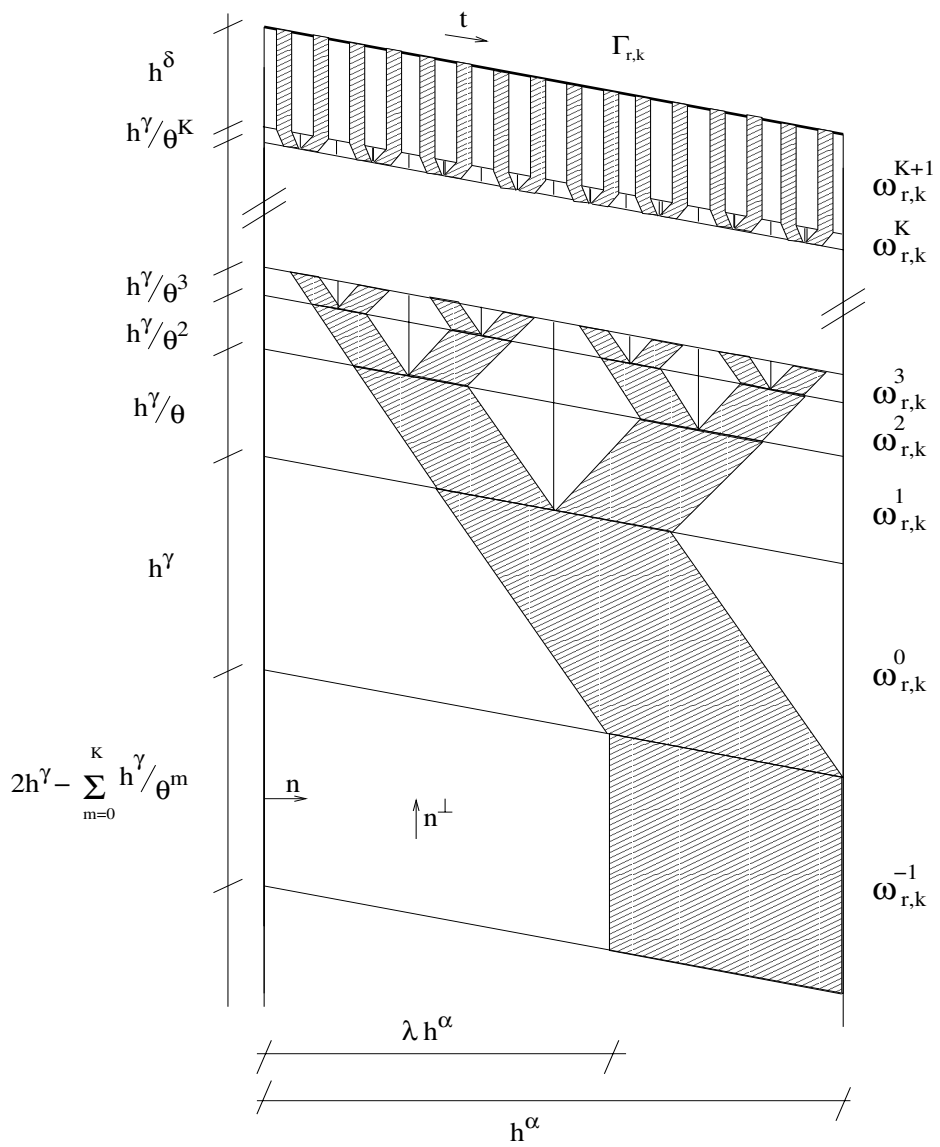

Fig. 3. Partition of $B_{r, k}$ and branching of the domains in which $\nabla \tilde{y}_{r, k}=F_{1}$ (blank) respectively $\nabla \tilde{y}_{r, k} \approx F_{2}$ (shaded). Note that there holds $\theta>2$

The assertions (a1), (a3), (a4), and (a5) follow from elementary manipulations. To prove (a2) we remark that for $I \subseteq(0,1)$ and

$$
G(x)=a \int_{0}^{u} \chi_{f(x)+I}(s / d) d s=d a \int_{0}^{u / d} \chi_{f(x)+I}(s) d s
$$

there holds

$$
\nabla G(x)=-d \chi_{f(x)+I}(u / d) a \otimes \nabla f(x) .
$$

Then the first assertion in (a2) follows since $\nabla d_{j}=-\theta^{j} n^{\perp} / h^{\gamma}$. To verify $(\theta / 2)^{j} h^{\alpha-\gamma} \leq C$ notice that $\alpha>\gamma$ and $K=0$ if $p=1$. If $p>1$ there holds, since $1 / s_{1} \leq p /(p-1)$ and $(\alpha-\delta) /(p-1)+\alpha-\gamma \geq 0$,

$$
\begin{aligned}
(\theta / 2)^{j} h^{\alpha-\gamma} & =\left(2^{1 / s_{1}-1}\right)^{j} h^{\alpha-\gamma} \leq\left(2^{K}\right)^{1 /(p-1)} h^{\alpha-\gamma} \\
& \leq C h^{(\alpha-\delta) /(p-1)+\alpha-\gamma} \leq C .
\end{aligned}
$$


We may now define a continuous mapping $\tilde{y}_{1} \in W^{1, \infty}(\omega)$ by setting, for $x \in \omega$,

$$
\tilde{y}_{1}(x):=\left\{\begin{array}{l}
\tilde{y}_{r, k}(x) \text { if } x \in B_{r, k} \\
\tilde{y}_{0}(x) \text { otherwise. }
\end{array}\right.
$$

(b) If $\Gamma_{r, k}$ fails to be affine but $\left.t\right|_{\Gamma_{r, k}} \mid n^{\perp}$ and $\ell_{n^{\perp}}\left(\omega_{k}\right) \geq 5 h^{\gamma}$ then $\omega_{k}$ can be divided into finitely many subdomains $\hat{\omega}_{k}^{\ell}$ such that $\Gamma_{r, k} \cap \partial \hat{\omega}_{k}^{\ell}$ is affine so that we have situation (a) for each of those subdomains after introducing a new coarse lamination of scale $\mathcal{O}\left(h^{\alpha}\right)$ in each $\hat{\omega}_{k}^{\ell}$. We assume that the lamination matches the lines that separate the domains $\hat{\omega}_{k}^{\ell}$. We then modify $\tilde{y}_{1}$ to a continuous function $\tilde{y}_{2} \in W^{1, \infty}(\omega)$ as in (a).

(c) Assume that there exists a subset of $\Gamma_{r, k}$ on which $t \| n^{\perp}$. Then, since $\omega$ is convex, $\Gamma_{r, k} \cap\left\{x \in \mathbb{R}^{2}: x \cdot n=k h^{\alpha}\right\}=\emptyset$ or $\Gamma_{r, k} \cap\left\{x \in \mathbb{R}^{2}\right.$ : $\left.x \cdot n=(k+1) h^{\alpha}\right\}=\emptyset$. Without loss of generality we will consider the latter case which is sketched in the left plot of Figure 4. Since we may assume $\ell_{n^{\perp}}\left(\omega_{k}\right) \geq 5 h^{\gamma}$ we may proceed as follows. We introduce a boundary region $\omega_{k}^{B L}$ of thickness $h^{\delta}$ as depicted in the left plot of Figure 4. In the remaining part of $\omega_{k}$ we introduce one lamination of scale $\mathcal{O}\left(h^{\alpha}\right)$ such that the lines separating $\omega_{k} \backslash \omega_{k}^{B L}$ from $\omega_{k-1}$ and $\omega_{k}^{B L}$ are matched by the laminates. We then define a branching of the laminate as in (a). Finally we modify $\tilde{y}_{2}$ to a function $\tilde{y}_{3} \in W^{1, \infty}(\omega)$ as in (a) and such that $\tilde{y}_{3}(x)=F x$ for all $x \in \omega_{k}^{B L}$.

(d) Assume now that $\ell_{n^{\perp}}\left(\omega_{k}\right) \leq 5 h^{\gamma}$ (so that we cannot define branchings as in (a) at both "ends" of $\left.\omega_{k}\right), \omega_{k \pm(m+1)}=\emptyset$ for some $m$. We may assume that the domains $\omega_{k+1}, \ldots, \omega_{k+m}$ (or $\left.\omega_{k-1}, \ldots, \omega_{k-m}\right)$ satisfy $\ell_{n^{\perp}}\left(\omega_{k+j}\right) \sim$ $\ell_{n^{\perp}}\left(\omega_{k+j+1}\right)+h^{\alpha}, j=1, \ldots, m-1$. We then define $\tilde{\omega}_{k}:=\cup_{j=0}^{m} \omega_{k+j}$ and partition $\tilde{\omega}_{k}$ as follows. Let $M:=\log _{2}\left(h^{\gamma-\delta}\right)+\overline{\bar{\varepsilon}}, M \in \mathbb{N}, \overline{\bar{\varepsilon}} \in[0,1]$, and define for $j=1, \ldots, M$,

$$
\hat{\omega}_{k}^{j}:=\left\{x \in \omega: k h^{\alpha}+\sum_{\ell=1}^{j-1} h^{\gamma} / 2^{\ell} \leq x \cdot n \leq k h^{\alpha}+\sum_{\ell=1}^{j} h^{\gamma} / 2^{\ell}\right\} .
$$

Since we may assume that $d_{n}\left(\tilde{\omega}_{k}\right)=\mathcal{O}\left(h^{\gamma}\right)$ (note that $\partial \omega$ is piecewise affine) we may also assume that

$$
\omega_{k}^{B L}:=\left\{x \in \omega: k h^{\alpha}+\sum_{\ell=1}^{M} h^{\gamma} / 2^{\ell} \leq x \cdot n\right\}
$$

is non-empty and satisfies $\ell_{n^{\perp}}\left(\omega_{k}^{B L}\right) \sim h^{\gamma} / 2^{M} \leq C h^{\delta}$ and $d_{n}\left(\omega_{k}^{B L}\right) \sim h^{\delta}$. The decomposition of $\tilde{\omega}_{k}$ is depicted in the right plot of Figure 4 . In each $\hat{\omega}_{k}^{j}, j=1, \ldots, M$, we introduce a lamination of scale $C h^{\alpha} / 2^{s_{2} j}$ with $s_{2}:=$ $\frac{\alpha-\delta}{\gamma-\delta} \in[0,1)$ and a constant $C>0$ so that the lines separating $\hat{\omega}_{k}^{j}$ from $\hat{\omega}_{k}^{j-1}$ 

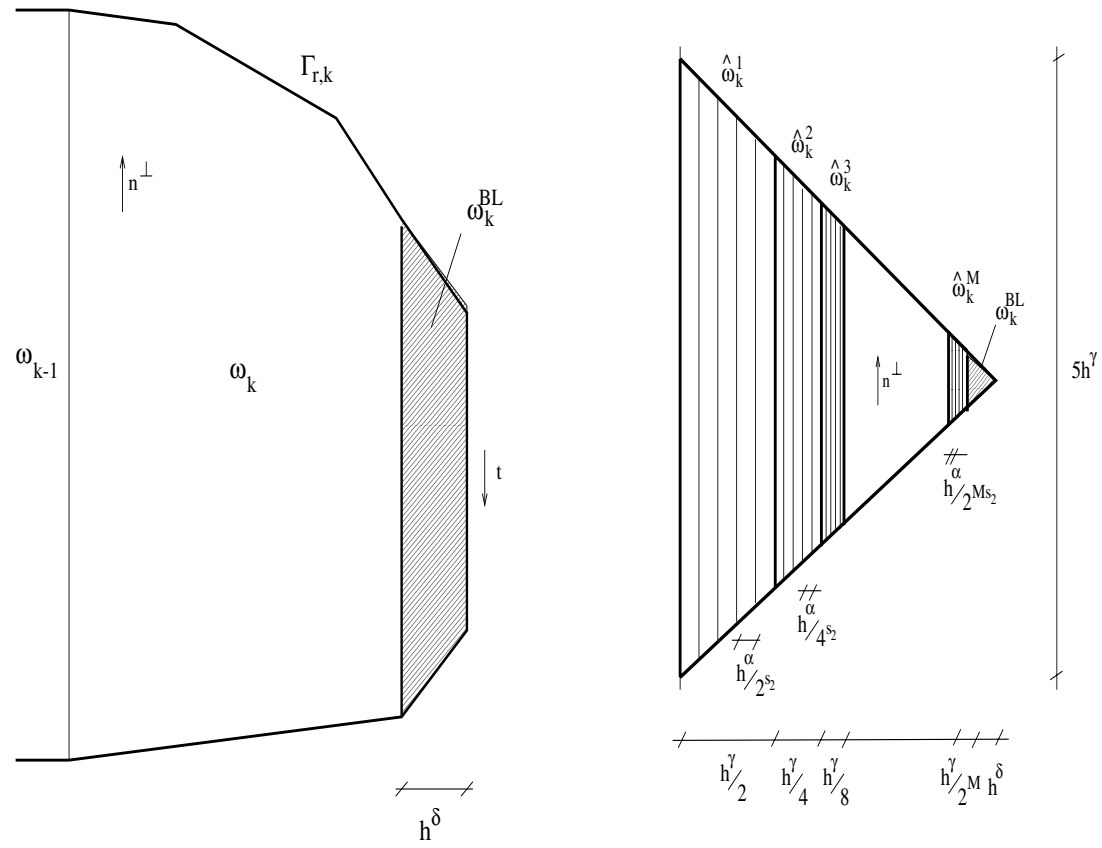

Fig. 4. Partitions of $\omega_{k}$ if $t \| n^{\perp}$ (left plot) and if $\ell_{n^{\perp}}\left(\omega_{k}\right) \leq 5 h^{\gamma}$ (right plot)

and $\hat{\omega}_{k}^{j+1}$ are matched by lamination interfaces. We then define a branching towards the boundary as in (a) (or (b)) but with scales $h^{\gamma} /\left(2^{j} \theta^{\ell}\right)$ and $h^{\alpha} / 2^{j s_{2}+\ell}, \ell=1, \ldots, K_{j}$, with $K_{j}=\log _{2}\left(2^{-j s_{2}} h^{\alpha-\delta}\right)+\varepsilon_{j}$, instead of $h^{\gamma} / 2^{\ell}$ and $h^{\alpha} / 2^{\ell}, \ell=1, \ldots, K$. Notice that the branching region refines a lamination of scale $\mathcal{O}\left(h^{\alpha} / 2^{s_{2} j}\right)$ to a lamination of scale $\mathcal{O}\left(h^{\alpha} / 2^{j s_{2}+K_{j}}\right)=\mathcal{O}\left(h^{\delta}\right)$. Finally we modify $\tilde{y}_{3}$ in $\tilde{\omega}_{k}$ to a function $\tilde{y}_{4} \in W^{1, \infty}(\omega)$ as above and such that $\tilde{y}_{4}(x)=F x$ for all $x \in \omega_{k}^{B L}$.

Step 3: Definition of the global continuous and discrete deformations. We now have to modify $\tilde{y}_{4}$ such that it satisfies the boundary conditions. Let $\rho \in C^{1}(\omega)$ satisfy $\rho=0$ in a boundary layer of thickness $\mathcal{O}(h), \rho=1$ outside a boundary layer of thickness $\mathcal{O}\left(h^{\delta}\right)$ and $|\nabla \rho(x)| \leq C / h^{\delta}$ for all $x \in \omega$. Define, for all $x \in \omega$,

$$
y(x)=(1-\rho(x)) F x+\rho(x) \tilde{y}_{4}(x) .
$$

Then, $y \in W^{1, \infty}(\omega)$ satisfies $y(x)=F x$ for all $x \in \partial \omega$ and we extend $y$ to $\cup \tau \backslash \omega$ by $F x$. Let $y_{h}:=\mathcal{I}_{h} y$ denote the nodal interpolant of $y$ which, by choice of $\rho$, also satisfies the boundary condition. There holds $\left.y_{h}\right|_{T}=\left.y\right|_{T}$ if $T \in \tau$ and $\left.\nabla y\right|_{T}$ is constant. Since we assume that $\tau$ is regular we also have that $\left\|\nabla \mathcal{I}_{h} v\right\|_{L^{\infty}(\cup \tau)} \leq C\|\nabla v\|_{L^{\infty}(\cup \tau)}$ for all $v \in W^{1, \infty}(\cup \tau) \cap C(\overline{\cup \tau})$. 
Step 4: Estimation of the energy of the discrete deformation. To estimate

$$
\int_{\omega} \operatorname{dist}\left(\nabla y_{h}(x),\left\{F_{1}, F_{2}\right\}\right)^{p} d x
$$

we divide the integral into several contributions.

I. For all $x \in \omega_{r, k}^{K+1}$ and $x \in \omega_{k}^{B L}$ (i.e., in a boundary layer of thickness $h^{\delta}$ ) we have, by choice of $\rho$,

$$
\begin{aligned}
|\nabla y(x)| & \leq\left|\left(F x-\tilde{y}_{4}(x)\right) \nabla \rho(x)\right|+\left|\rho(x)\left(F+\nabla \tilde{y}_{4}(x)\right)\right| \\
& \leq C\left(h^{-\delta}\left|\left(F x-\tilde{y}_{4}(x)\right)\right|+1\right),
\end{aligned}
$$

hence

$$
\begin{aligned}
\operatorname{dist}\left(\nabla y_{h}(x),\left\{F_{1}, F_{2}\right\}\right) & \leq\left|\nabla y_{h}(x)-\nabla y(x)\right|+|\nabla y(x)|+\min _{j=1,2}\left|F_{j}\right| \\
& \leq C\left(1+h^{-\delta}\left|\left(F x-\tilde{y}_{4}(x)\right)\right|\right) .
\end{aligned}
$$

Since the union of all domains $\omega_{r, k}^{K+1}$ and $\omega_{k}^{B L}$ defines a strip along $\partial \omega$ of width $h^{\delta}$, property (a4) ensures $\left|F x-\tilde{y}_{4}(x)\right| \leq h^{\delta}$ while $\tilde{y}(x)=F x$ in $\omega_{k}^{B L}$ as defined in (c) and (d) of Step 2 so that

$$
\int_{\left(\cup \omega_{r, k}^{K+1}\right) \cup\left(\cup \omega_{k}^{B L}\right)} \operatorname{dist}\left(\nabla y_{h}(x),\left\{F_{1}, F_{2}\right\}\right)^{p} d x \leq C|\partial \omega| h^{\delta} .
$$

II. In the branchings defined in (a) of Step 2 the energy in $\omega_{r, k}^{0} \cup \ldots \cup \omega_{r, k}^{K}$ can be estimated as follows. In a distance $\geq h$ from the line segments in whose neighbourhood $\nabla y$ is not constant there holds by (a2) of Step 2, $\nabla y_{h}(x)=\nabla \tilde{y}_{r, k} \in\left\{F_{1}, F_{2}\right\}+\mathcal{O}\left(\theta^{j} h^{\alpha-\gamma} / 2^{j}\right)$. In an $h$-neighbourhood of the line segments, there holds, (cf. (a2) in Step 2) $\left|\nabla y_{h}(x)-F_{j}\right| \leq C$, $j=1,2$. The $K$ interfaces separating domains $\omega_{r, k}^{j}$ have a maximal length $h^{\alpha}$ and contribute to the energy in an $h$-neighbourhood. In each of the domains $\omega_{r, k}^{j}, j=0, \ldots, K$ there are $2^{j}$ line segments of length $h^{\gamma} / \theta^{j}$ which contribute to the energy in an $h$-neighbourhood. Since $\gamma<\alpha$ we may estimate $K h^{1+\alpha}<C h^{1+\gamma}$. For one domain $B_{r, k}$ we thus have, using $\theta>2$ and $\theta^{p-1} / 2^{p}<1$

$$
\begin{aligned}
& \int_{B_{r, k}} \operatorname{dist}\left(\nabla y_{h}(x),\left\{F_{1}, F_{2}\right\}\right)^{p} d x \\
& \quad \leq C \sum_{j=0}^{K}\left(\left(h^{\alpha+\gamma} / \theta^{j}\right)\left(\theta^{j} h^{\alpha-\gamma} / 2^{j}\right)^{p}+2^{j} h h^{\gamma} / \theta^{j}+h h^{\alpha}\right) \\
& \quad \leq C\left(h^{(p+1) \alpha-(p-1) \gamma}+h^{1+\gamma}\right) .
\end{aligned}
$$

Since there are at most $C d_{n}(\omega) h^{-\alpha}$ many domains $B_{r, k}$ as in (a) and since $\gamma \leq \alpha$ it follows that

$$
\int_{\cup B_{r, k}} \operatorname{dist}\left(\nabla y_{h}(x),\left\{F_{1}, F_{2}\right\}\right)^{p} d x \leq C d_{n}(\omega)\left(h^{p \alpha-(p-1) \gamma}+h^{1+\gamma-\alpha}\right) .
$$


III. There are at most $d_{n}(\omega) h^{-\alpha}$ many line segments in the interior of $\omega$ (i.e., away from the branching regions) that separate domains $\omega_{k}^{1}$ and $\omega_{k}^{2}$. In their $h$-neighbourhood $\nabla y$ is non-constant. Since those line segments have a maximal length $\ell_{n^{\perp}}(\omega)$ they lead to an energy contribution

$$
\int_{\left\{x \in \omega: x \notin B_{r, k}\right\} \cup\left(\cup \omega_{r, k}^{-1}\right)} \operatorname{dist}\left(\nabla y_{h}(x),\left\{F_{1}, F_{2}\right\}\right)^{p} d x \leq C d_{n}(\omega) \ell_{n^{\perp}}(\omega) h^{1-\alpha} .
$$

IV. The boundary regions defined in (c) of Step 2 can be estimated as in I.-III. Note that the number of such domains is independent of $h$.

V. Assume now the situation from (d) of Step 2. In each $\hat{\omega}_{k}^{j}, j=1, \ldots, M$, there are $h^{\gamma} h^{-\alpha} 2^{j\left(s_{2}-1\right)}$ many line segments of length $h^{\gamma} / 2^{j}$ which contribute to the energy in an $h$-neighbourhood. Moreover, from the branchings in $\hat{\omega}_{k}^{j}$ we obtain a contribution (cf. II.)

$$
h^{\gamma-\alpha} 2^{j\left(s_{2}-1\right)} h^{p(\alpha-\gamma)} 2^{p j\left(1-s_{2}\right)} h^{\gamma+\alpha} 2^{-j\left(s_{2}+1\right)} .
$$

Note that by assumption we have $1>s_{2} \geq(p-1) / p$ so that $p\left(1-s_{2}\right)-1 \leq 0$. The line segments in those branching regions lead to an energy

$$
h^{\gamma-\alpha} 2^{j\left(s_{2}-1\right)} h^{1+\gamma} 2^{-j} .
$$

The summation over the domains $\hat{\omega}_{k}^{1}, \ldots, \hat{\omega}_{k}^{M}$ and noting that the boundary layer $\omega_{k}^{B L}$ contributes an amount $|\partial \omega| h^{\delta}$ shows

$$
\begin{aligned}
\int_{\tilde{\omega}_{k}} & \operatorname{dist}\left(\nabla y_{h}(x),\left\{F_{1}, F_{2}\right\}\right)^{p} d x \leq C|\partial \omega| h^{\delta}+C \sum_{j=1}^{M} h^{\gamma-\alpha} 2^{j\left(s_{2}-1\right)} \\
& \times\left(h^{p(\alpha-\gamma)} 2^{p j\left(1-s_{2}\right)} h^{\gamma+\alpha} 2^{-\left(1+s_{2}\right) j}+h^{1+\gamma} 2^{-j}+h^{1+\gamma} 2^{-j}\right) \\
\leq & C|\partial \omega| h^{\delta}+C h^{\gamma}\left(h^{p \alpha-(p-1) \gamma}+h^{1+\gamma-\alpha}+h^{1+\gamma-\alpha}\right) .
\end{aligned}
$$

Since $h^{\gamma} \leq C d_{n}(\omega)$ and $h^{\gamma} \leq C \ell_{n^{\perp}}(\omega)$ the right-hand side is bounded by the same quantities as in I., II., and III.

The summation of the contributions in I.-V. proves the assertion of the lemma.

\section{Application to a three-well problem}

The explicit dependence of the energy estimate in Lemma 2.1 on various quantities related to $\omega$ allows for an iterative application. Layers within layers without branching (or transition regions) are depicted in Figure 5. We will proceed analogously but with appropriate branching at the boundary and at interfaces between different variants. 


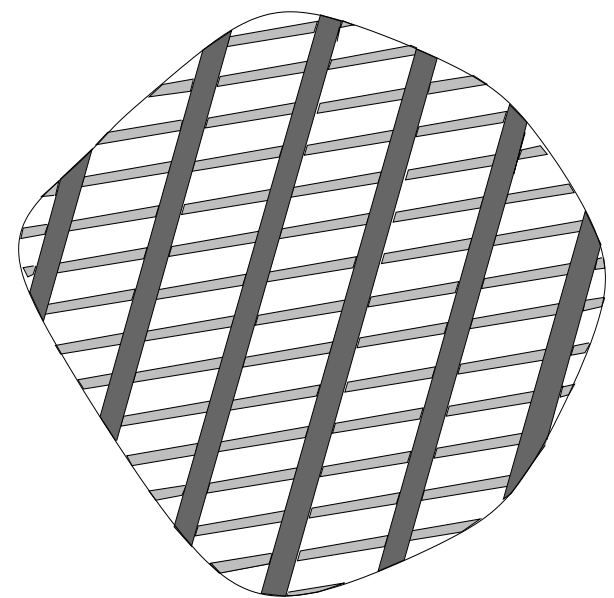

Fig. 5. Iterated lamination in $\Omega$ (without branching or transition layers) using three different gradients

Theorem 3.1 Suppose that $F=\lambda\left(\rho F_{11}+(1-\rho) F_{12}\right)+(1-\lambda) F_{2}$ for $F_{11}, F_{12}, F_{2} \in \mathbb{R}^{2 \times 2}$ and $\rho, \lambda \in(0,1)$. Moreover, assume that $F_{1}-F_{2}=$ $a \otimes n$ for $a, n \in \mathbb{R}^{2}$ and $|n|=1$, where $F_{1}=\rho F_{11}+(1-\rho) F_{12}$ such that there exist $b, m \in \mathbb{R}^{2},|m|=1, m \nVdash n$, with $F_{11}-F_{12}=b \otimes m$. If $h$ is small enough there exists $y_{h} \in \mathcal{S}^{1}(\mathcal{T})^{2}$ satisfying $y_{h}(x)=F x$ for all $x \in \partial \Omega$ such that there holds

$$
\int_{\Omega} \operatorname{dist}\left(\nabla y_{h}(x),\left\{F_{11}, F_{12}, F_{2}\right\}\right)^{p} d x \leq C h^{p /(p+2)} .
$$

Proof. We start as in the proof of Lemma 2.1 and define with $F_{1}, F_{2},(\alpha, \gamma, \delta)$ $=\left(\alpha_{1}, \gamma_{1}, \delta_{1}\right)$, and $\omega=\Omega$ a function $\tilde{y}$ by performing Steps 1-3 in the proof of Lemma 2.1. To each subdomain $\omega \subseteq \Omega$ outside the boundary layers $\omega_{k}^{B L}$ or $\omega_{r, k}^{K+1}$, and such that $\left.\nabla \tilde{y}\right|_{\omega}=F_{1}$, we want to apply Lemma 2.1 with appropriate scales $\left(\alpha_{2}, \gamma_{2}, \delta_{2}\right)$ (if necessary choosing the coarsest scale of the branchings in $\omega$ as $\left(h^{\alpha_{2}} / 2^{s j+k}, h^{\gamma_{2}} /\left(\theta^{j} 2^{k}\right)\right)$ for some $\left.j \geq 0\right), F_{11}, F_{12}$, and $\rho$. This defines a mapping $\left.y_{h}\right|_{\omega}$ satisfying $y_{h}(x)=F_{1} x$ for all $x \in \partial \omega$. Since $\tilde{y}(x)=F_{1} x+a$ for all $x \in \omega$ and some $a \in \mathbb{R}^{2}$ the mapping

$$
\tilde{\tilde{y}}(x)=\left\{\begin{array}{l}
y_{h}(x)+a \text { for } x \in \omega, \\
\tilde{y}(x) \quad \text { for } x \in \Omega \backslash \omega,
\end{array}\right.
$$

is continuous. The nodal interpolant of $\tilde{\tilde{y}}$ then satisfies $\nabla \mathcal{I}_{h} \tilde{\tilde{y}}=\nabla y_{h}$ in $\omega \backslash\{x \in \omega: \operatorname{dist}(x, \partial \omega)>C h\}$. To estimate the energy of this mapping, we have to give an upper bound for the energy coming from those subdomains in which we modified $\tilde{y}$. From the part of $\Omega$ that we do not modify we get the contributions of Lemma 2.1 (with exponents $\left(\alpha_{1}, \gamma_{1}, \delta_{1}\right)$ and domain $\Omega$ ). We now estimate the energy coming from subdomains $\omega$ as above. 
Step 1: Domains $\omega$ away from the branchings. The mapping $\tilde{y}$ in the proof of Lemma 2.1 defines at most $d_{n}(\Omega) h^{-\alpha_{1}}$ many domains $\omega_{k}^{1}$ such that $d_{m}\left(\omega_{k}^{1}\right) \leq$ $C$ and $\ell_{m \perp}\left(\omega_{k}^{1}\right) \leq C h^{\alpha_{1}}$ in which $\nabla \tilde{y}=F_{1}$. Lemma 2.1 then defines for each such domain $\omega_{k}^{1}$ a function $\left.y_{h}\right|_{\omega_{k}^{1}}$, such that, for $\alpha_{1} \leq \gamma_{2} \leq \alpha_{2} \leq \delta_{2}$, there holds

$$
\begin{aligned}
& \int_{\cup \omega_{k}^{1}} \operatorname{dist}\left(\nabla y_{h}(x),\left\{F_{11}, F_{12}\right\}\right)^{p} d x \leq C h^{-\alpha_{1}} \max \left\{d_{m}\left(\omega_{k}^{1}\right),|\partial \omega|\right\} \\
& \quad \times\left(h^{\delta_{2}}+h^{p \alpha_{2}-(p-1) \gamma_{2}}+h^{1+\gamma_{2}-\alpha_{2}}+h^{1-\alpha_{2}+\alpha_{1}}\right) .
\end{aligned}
$$

Step 2: Domains in branching regions. In the branchings close to the boundary of $\Omega$ there are domains $\omega_{r, k}^{j, \ell}, j=0, \ldots, K$, with $K=\log _{2}\left(h^{\alpha_{1}-\delta_{1}}\right)+\bar{\varepsilon}$ and $\ell=1, \ldots, L_{j} \leq 3 \cdot 2^{j}$, in which $\nabla \tilde{y}=F_{1}$ and $d_{m}\left(\omega_{r, k}^{j, \ell}\right) \leq C h^{\gamma_{1}} / \theta^{j}$, $\ell_{m^{\perp}}\left(\omega_{r, k}^{j, \ell}\right) \leq C h^{\alpha_{1}} / 2^{j}$ and $\left|\partial \omega_{r, k}^{j, \ell}\right| \leq C h^{\gamma_{1}} / \theta^{j}$. To such domains we apply Lemma 2.1 with scales $\left(h^{\alpha_{2}} / 2^{j}, h^{\gamma_{2}} / \theta^{j}, h^{\delta_{2}}\right)$. We have to ensure that $h^{\alpha_{2}} / 2^{j} \geq C h^{\delta_{2}}, j=1, \ldots, K$. Since $2^{K} \leq C h^{\alpha_{1}-\delta_{1}}$ this is guaranteed if $\alpha_{1}-\delta_{1}=\alpha_{2}-\delta_{2}$. We then obtain mappings $\left.y_{h}\right|_{\omega_{r, k}^{j, \ell}}$ such that

$$
\begin{aligned}
& \int_{\omega_{r, k}^{j, \ell}} \operatorname{dist}\left(\nabla y_{h}(x),\left\{F_{11}, F_{12}\right\}\right)^{p} d x \leq C h^{\gamma_{1}} / \theta^{j} \\
& \quad \times\left(h^{\delta_{2}}+h^{p \alpha_{2}-(p-1) \gamma_{2}}+h^{1+\gamma_{2}-\alpha_{2}}+h^{1-\alpha_{2}+\alpha_{1}}\right)
\end{aligned}
$$

where we used $\theta>2$ and $\theta^{p-1} / 2^{p}<1$. The summation of all such domains shows, since there are at most $d_{n}(\Omega) h^{-\alpha_{1}}$ many domains $B_{r, k}$, in which branchings between $F_{1}$ and $F_{2}$ are needed,

$$
\begin{aligned}
& \int_{\cup \omega_{r, k}^{j, \ell}} \operatorname{dist}\left(\nabla y_{h}(x),\left\{F_{11}, F_{12}\right\}\right)^{p} d x \leq C h^{-\alpha_{1}} \sum_{j=0}^{K} 2^{j+1} h^{\gamma_{1}} / \theta^{j} \\
& \times\left(h^{\delta_{2}}+h^{p \alpha_{2}-(p-1) \gamma_{2}}+h^{1+\gamma_{2}-\alpha_{2}}+h^{1-\alpha_{2}+\alpha_{1}}\right) \\
& \quad \leq C h^{\gamma_{1}-\alpha_{1}}\left(h^{\delta_{2}}+h^{p \alpha_{2}-(p-1) \gamma_{2}}+h^{1+\gamma_{2}-\alpha_{2}}+h^{1-\alpha_{2}+\alpha_{1}}\right) .
\end{aligned}
$$

Step 3: Domains within corner domains. We now estimate the energy steming from replacing a gradient $F_{1}$ by a laminate in corner domains as considered in (d) in the proof of Lemma 2.1. Here, a domain $\omega$ in which a gradient equals $F_{1}$ satisfies

$$
|\partial \omega| \leq C h^{\gamma_{1}} /\left(2^{j} \theta^{k}\right), \quad d_{m}(\omega) \leq C h^{\gamma_{1}} /\left(2^{j} \theta^{k}\right)
$$

and

$$
\ell_{m^{\perp}}(\omega) \leq C h^{\alpha_{1}} / 2^{s_{2} j+k}
$$


for $j=1, \ldots, M \leq \log _{2}\left(h^{\gamma_{1}-\delta_{1}}\right)$ and $k=1, \ldots, K_{j} \leq \log _{2}\left(2^{-s_{2} j} h^{\alpha_{1}-\delta_{1}}\right)$. The lamination in $\omega$ using gradients $F_{11}$ and $F_{12}$ is then on a scale $h^{\gamma_{2}} /\left(2^{j} \theta^{k}\right)$ and $h^{\alpha_{2}} / 2^{s_{2} j+k}$ and the branching starts on that scale and refines the lamination by successively dividing $h^{\alpha_{2}} / 2^{s_{2} j+k}$ and $h^{\gamma_{2}} /\left(2^{j} \theta^{k}\right)$ by 2 and $\theta$, respectively, until $h^{\alpha_{2}} / 2^{s_{2} j+k+m}=h^{\delta_{2}}$. Since $p-p s_{2}-1 \leq 0$ and $s_{2}=\frac{\delta_{1}-\alpha_{1}}{\delta_{1}-\gamma_{1}}<1$ we obtain a contribution

$$
\begin{aligned}
\int_{\omega} & \operatorname{dist}\left(\nabla y_{h}(x),\left\{F_{11}, F_{12}\right\}\right)^{p} d x \\
\leq & C h^{\gamma_{1}} /\left(2^{j} \theta^{k}\right)\left(h^{\delta_{2}}+h^{p \alpha_{2}-(p-1) \gamma_{2}} 2^{-j p s_{2}} \theta^{j(p-1)}\right. \\
& \left.\quad+h^{1+\gamma_{2}-\alpha_{2}} 2^{j s_{2}} \theta^{-j}+h^{1-\alpha_{2}+\alpha_{1}}\right) \\
\leq & C h^{\gamma_{1}} /\left(2^{j} \theta^{k}\right)\left(h^{\delta_{2}}+h^{p \alpha_{2}-(p-1) \gamma_{2}}+h^{1+\gamma_{2}-\alpha_{2}}+h^{1-\alpha_{2}+\alpha_{1}}\right) .
\end{aligned}
$$

We now have to sum all such domains $\omega$. Noting that in each domain $\hat{\omega}_{k}^{j}$ there are $\frac{h^{\gamma_{1}}}{2^{j}} \frac{2^{s j}}{h^{\alpha_{1}}}$ many branching regions and laminates we find

$$
\begin{aligned}
& \int_{\cup \omega} \operatorname{dist}\left(\nabla y_{h}(x),\left\{F_{11}, F_{12}\right\}\right)^{p} d x \leq C \sum_{j=1}^{M} \frac{h^{\gamma_{1}}}{2^{j}} \frac{2^{s_{2} j}}{h^{\alpha_{1}}} \\
& \times\left(\sum_{k=0}^{K_{j}-j} 2^{k} \frac{h^{\gamma_{1}}}{2^{j} \theta^{k}}\left(h^{\delta_{2}}+h^{p \alpha_{2}-(p-1) \gamma_{2}}+h^{1+\gamma_{2}-\alpha_{2}}+h^{1-\alpha_{2}+\alpha_{1}}\right)\right) \\
& \leq C h^{\gamma_{1}-\alpha_{1}}\left(h^{\delta_{2}}+h^{p \alpha_{2}-(p-1) \gamma_{2}}+h^{1+\gamma_{2}-\alpha_{2}}+h^{1-\alpha_{2}+\alpha_{1}}\right) .
\end{aligned}
$$

Step 4: Estimation of the total energy. The two lamination processes lead to a total energy

$$
\begin{aligned}
& \int_{\Omega} \operatorname{dist}\left(\nabla y_{h}(x),\left\{F_{11}, F_{12}, F_{2}\right\}\right)^{p} d x \\
& \leq C\left(h^{\gamma_{1}-\alpha_{1}}\left(h^{\delta_{2}}+h^{p \alpha_{2}-(p-1) \gamma_{2}}+h^{1+\gamma_{2}-\alpha_{2}}+h^{1-\alpha_{2}+\alpha_{1}}\right)\right. \\
& \left.\quad+h^{\delta_{1}}+h^{p \alpha_{1}-(p-1) \gamma_{1}}+h^{1+\gamma_{1}-\alpha_{1}}+h^{1-\alpha_{1}}\right) .
\end{aligned}
$$

For $j=1,2$ we choose $\alpha_{j}=j /(p+2), \gamma_{j}=(j-1) /(p+2)$, and $\delta_{j}=(p+j-1) /(p+2)$ and check that the assumptions on $s_{1}$ (which defines $\theta), s_{2}$, and $\alpha_{2}-\delta_{2}=\alpha_{1}-\delta_{1}$ are satisfied to conclude the proof.

Remark 3.1 In the previous proof we chose the first lamination such that we have gradients $F_{1}$ in the branching regions and then replaced $F_{1}$ by another lamination. Some care has to be taken if one also wants to replace $F_{2}$ since in the branching regions the deformation gradient is not exactly equal to $F_{2}$. Instead, one has a gradient $F_{2}+\tilde{F}_{2}$ with $\tilde{F}_{2}=\mathcal{O}\left(\theta^{j} h^{\alpha_{1}-\gamma_{1}} / 2^{j}\right)$. One may then replace $F_{2}$ by a lamination and add $\tilde{F}_{2}$ after that process again. The resulting energy is then estimated with the help of the triangle inequality to obtain the same bound. 


\section{Application to higher-order laminates}

We now perform the iterative lamination process for arbitrary lamination orders. We will assume that the boundary data lies in the lamination convex hull of $\left\{F_{1}, \ldots, F_{N}\right\}$ in the following sense.

Definition 4.1 The homogeneous deformation $F \in \mathbb{R}^{n \times n}$ lies in the lamination convex hull of $\left\{F_{1}, \ldots, F_{N}\right\}$ if there exist a positive integer $L, E_{j, k} \in$ $\mathbb{R}^{n \times n}, \varrho_{j, k} \in[0,1], a_{j, k} \in \mathbb{R}^{n}, n_{j, k} \in \mathbb{R}^{n},\left|n_{j, k}\right|=1, j=0,1,2, \ldots, L$, $k=1,2, \ldots, 2^{j}$, such that $F=E_{0,1}$ and

$$
\begin{aligned}
& E_{j, k}=\varrho_{j, k} E_{j+1,2 k-1}+\left(1-\varrho_{j, k}\right) E_{j+1,2 k}, \\
& E_{j+1,2 k}-E_{j+1,2 k-1}=a_{j, k} \otimes n_{j, k}, \\
& E_{L, k} \in\left\{F_{1}, \ldots, F_{N}\right\} .
\end{aligned}
$$

To apply Lemma 2.1 we will also assume that two successive normals in the construction of the boundary data $F$ in the lamination convex hull of $\left\{F_{1}, \ldots, F_{N}\right\}$ are not parallel.

Theorem 4.1 Assume that $F \in \mathbb{R}^{2 \times 2}$ lies in the lamination convex hull of $\left\{F_{1}, \ldots, F_{N}\right\}$ and assume that if $F$ is constructed as in Definition 4.1 for $j=0,1, \ldots, L-1, k=1,2, \ldots, 2^{j}$, the normal $n_{j, k}$ is neither parallel to $n_{j+1,2 k-1}$ nor to $n_{j+1,2 k}$. If $h$ is small enough there exists $y_{h} \in \mathcal{S}^{1}(\mathcal{T})^{2}$ satisfying $y_{h}(x)=F x$ for all $x \in \partial \Omega$ and such that

$$
\inf _{v_{h} \in \mathcal{A}_{h}} I\left(v_{h}\right) \leq C h^{p /(p+L)} .
$$

Remark 4.1 Improved estimates for the approximation of volume fractions and Young measure support as well as for convergence of $v_{h}$ in $L^{2}$ follow from the theorem. We refer to $[20,12]$.

Proof. As in the proof of Theorem 3.1 we define a deformation $y_{h}$ by successively replacing a deformation gradient $E_{j, k}$ by $E_{j+1,2 k-1}$ and $E_{j+1,2 k}$ with the help of Lemma 2.1. If we ensure $\alpha_{m}-\delta_{m}=\alpha_{1}-\delta_{1}$ and $\gamma_{m}-\delta_{m}=\gamma_{1}-\delta_{1}$ for $m=1, \ldots, L$ we may set $s_{2}=\frac{\delta_{1}-\alpha_{1}}{\delta_{1}-\gamma_{1}} \in[0,1]$ and assume that in a domain $\omega$ with $d_{n_{L-1, \ell}}(\omega) \leq C h^{\alpha_{L-2}}$ or $d_{n_{L-1, \ell}}(\omega) \leq C h^{\gamma_{L-1}} /\left(2^{k_{1}} \theta^{k_{2}}\right)$ and $\ell_{n_{L-1, \ell}}(\omega) \leq$ $C h^{\alpha_{L-1}} / 2^{k^{\prime} s+k^{\prime \prime}}$ for $k_{1}+k_{2}=k^{\prime}+k^{\prime \prime}=k$, where $k \leq \log _{2}\left(h^{\alpha_{L-1}-\delta_{L-1}}\right)$, we replaced a gradient $E_{L-1, \ell}$ by a lamination and branching of $E_{L, 2 \ell}$ and $E_{L, 2 \ell+1}$. Suppose (and check later) that $h^{\alpha_{L-2}} \sim h^{\gamma_{L-1}}$ so that we only have to consider the latter case and include $d_{n_{L-1, \ell}}(\omega) \leq C h^{\alpha_{L-2}}$ in the subcase $k=0$. The domain $\omega$ then contributes to the total energy the amount (cf. the proof of Theorem 3.1)

$$
\begin{gathered}
\int_{\omega} \operatorname{dist}\left(\nabla y_{h}(x),\left\{E_{L, 2 k}, E_{L, 2 k-1}\right\}\right)^{p} d x \leq C h^{\gamma_{L-1}} /\left(2^{k_{1}} \theta^{k_{2}}\right) \\
\times\left(h^{\delta_{L}}+h^{p \alpha_{L}-(p-1) \gamma_{L}}+h^{1+\gamma_{L}-\alpha_{L}}+h^{1-\alpha_{L}+\alpha_{L-1}}\right) .
\end{gathered}
$$


We now have to count how many such domains $\omega$ there are. Let us set $\alpha_{-1}=$ $\alpha_{0}=\gamma_{0}=\delta_{0}:=0$ and $\tilde{\gamma}_{j}:=\min \left\{\gamma_{j}, \alpha_{j-1}\right\}, j=0, \ldots, L$. After the first lamination there are in laminates and branchings as defined in (a) in the proof of Lemma 2.1

$$
h^{\tilde{\gamma_{0}-\alpha_{1}}} 2^{j_{1}}
$$

many domains $\omega$ with $d_{n_{1,1}}(\omega) \leq C h^{\gamma_{1}} / \theta^{j_{1}}$. In corner domains there are at most (note $s_{2}<1$ )

$$
\sum_{\ell=0}^{M} \frac{h^{\gamma_{1}}}{2^{\ell}} \frac{2^{s_{2} \ell}}{h^{\alpha_{1}}} 2^{j_{1}} \leq C h^{\tilde{\gamma}_{0}-\alpha_{1}} 2^{j_{1}}
$$

many domains $\omega$ with $d_{n_{1,2}}(\omega) \leq C h^{\gamma_{1}} /\left(2^{s_{2} k_{1}} \theta^{j_{1}}\right)$ for some $k_{1}=0, \ldots, K-j_{1}$. Since in those domains we use a lamination of scale $h^{\alpha_{2}} / 2^{s_{2} k_{1}+j_{1}}$ and a branching starting on scales $h^{\alpha_{2}} / 2^{k_{1}+j_{1}}, h^{\gamma_{2}} /\left(2^{k_{1}} \theta^{j_{1}}\right)$ while the corner domains themselves start on a scale $h^{\gamma_{2}} / 2^{k_{1}}$, we have

$$
h^{\tilde{\gamma_{0}-\alpha_{1}}} 2^{j_{1}}\left(h^{\tilde{\gamma_{1}}-\alpha_{2}} 2^{j_{2}}+\sum_{\ell=0}^{K} \frac{h^{\gamma_{2}}}{2^{\ell}} \frac{2^{s_{2} \ell}}{h^{\alpha_{2}}} 2^{j_{2}}\right) \leq C h^{\tilde{\gamma_{0}}-\alpha_{1}} 2^{j_{1}} h^{\tilde{\gamma_{1}}-\alpha_{2}} 2^{j_{2}}
$$

many domains $\omega$ with $d_{n_{2, m}}(\omega) \leq C h^{\gamma_{2}} /\left(2^{k_{1}} \theta^{j_{1}+j_{2}}\right)$ for $m=1, \ldots, 4$ and $k_{1} \leq K-j_{1}-j_{2}$ after the second lamination. Iterating the argumentation we find that there are

$$
\Pi_{\ell=1}^{L-1}\left(h^{\tilde{\gamma}_{\ell-1}-\alpha_{\ell}} 2^{j_{\ell}}\right)=2^{j_{1}+j_{2}+\ldots+j_{L-1}}\left(\Pi_{\ell=1}^{L-1} h^{\gamma_{\ell-1}-\alpha_{\ell}}\right)
$$

many domains $\omega$ in which we have a gradient on level $L-1$ and such that $d_{n_{L-1, m}}(\omega) \leq C h^{\gamma_{L-1}} /\left(2^{k_{1}} \theta^{j_{1}+j_{2}+\ldots+j_{L-1}}\right)$ for $m=1, \ldots, 2^{L-1}$ and $k_{1} \leq$ $K-j_{1}-j_{2}-\ldots-j_{L-1}$. Summing the energy contributions (4.1) for $j_{1}+$ $j_{2}+\ldots+j_{L-1}=k_{2}=0, \ldots, K$ and allowing $k_{1}$ to range between 0 and $K-k_{2}$ we find

$$
\begin{aligned}
\int_{\cup \omega} \operatorname{dist}\left(\nabla y_{h}(x),\left\{E_{L, 2 k}, E_{L, 2 k-1}\right\}\right)^{p} d x \\
\leq C \sum_{k_{2}=0}^{K} \sum_{k_{1}=0}^{K-k_{2}} \sum_{j_{1}+j_{2}+\ldots+j_{L-1}=k_{2}} 2^{j_{1}+j_{2}+\ldots+j_{L-1}}\left(\Pi_{\ell=1}^{L-1} h^{\gamma_{\ell-1}-\alpha_{\ell}}\right) \\
\quad \times\left(h^{\gamma_{L-1}} /\left(2^{k_{1}} \theta^{k_{2}}\right)\right)\left(h^{\delta_{L}}+h^{p \alpha_{L}-(p-1) \gamma_{L}}+h^{1+\gamma_{L}-\alpha_{L}}+h^{1-\alpha_{L}+\alpha_{L-1}}\right) .
\end{aligned}
$$

Using that $\sum_{j_{1}+j_{2}+\ldots+j_{L-1}=k_{2}} 1 \leq k_{2}^{L-1}, \sum_{k_{1}=0}^{K-k_{2}} 2^{-k_{1}} \leq C$, and, since $\theta>2$, $\sum_{k_{2}=0}^{K} k_{2}^{L-1}(2 / \theta)^{k_{2}} \leq C$ we deduce

$$
\begin{aligned}
& \int_{\cup \omega} \operatorname{dist}\left(\nabla y_{h}(x),\left\{E_{L, 2 k}, E_{L, 2 k-1}\right\}\right)^{p} d x \leq C\left(\Pi_{\ell=1}^{L-1} h^{\gamma_{\ell-1}-\alpha_{\ell}}\right) h^{\gamma_{L-1}} \\
& \times\left(h^{\delta_{L}}+h^{p \alpha_{L}-(p-1) \gamma_{L}}+h^{1+\gamma_{L}-\alpha_{L}}+h^{1-\alpha_{L}+\alpha_{L-1}}\right) .
\end{aligned}
$$


Summing the contribution from each lamination step (since the binary tree might have a local depth $\leq L$ ) we obtain the following bound on the energy

$$
\begin{aligned}
I\left(y_{h}\right) \leq & C \sum_{k=1}^{L}\left(\Pi_{\ell=1}^{k-1} h^{\tilde{\gamma}_{\ell-1}-\alpha_{\ell}}\right) \\
& \times h^{\gamma_{k-1}}\left(h^{\delta_{k}}+h^{p \alpha_{k}-(p-1) \gamma_{k}}+h^{1+\gamma_{k}-\alpha_{k}}+h^{1-\alpha_{k}+\alpha_{k-1}}\right) .
\end{aligned}
$$

For $j=1, \ldots, L$ we choose $\alpha_{j}=j /(p+L), \gamma_{j}=(j-1) /(p+L)$, and $\delta_{j}=(p+j-1) /(p+L)$ so that

$$
\left(\Pi_{\ell=1}^{k-1} h^{\tilde{\gamma}_{\ell-1}-\alpha_{\ell}}\right) h^{\gamma_{k-1}}=h^{-\alpha_{k-1}} .
$$

We then have $\inf _{v_{h} \in \mathcal{A}_{h}} I\left(v_{h}\right) \leq C h^{p /(p+L)}$.

Acknowledgements. The authors wish to thank the anonymous referees for their suggestions to improve the results of this paper. The authors acknowledge stimulating discussions with Professor C. Carstensen. Both authors are thankful for financial support by the German Research Foundation (DFG) within the Graduiertenkolleg "Effiziente Algorithmen und Mehrskalenmethoden".

\section{References}

1. Ball, J.M., James, R.D.: Fine phase mixtures as minimizers of energy. Arch. Rat. Mech. Anal. 100, 13-52 (1987)

2. Ball, J.M., James, R.D.: Proposed experimental tests of a theory of fine microstructure and the two-well problem. Phil. Trans. R. Soc. Lond. A 338, 389-450 (1992)

3. Bartels, S.: Numerical analysis of some non-convex variational problems. PhDThesis, Universität Kiel, 2001. Available at http://e-diss.uni-kiel.de/math-nat.html

4. Belgacem, H.B., Conti, S., DeSimone, A., Müller, S.: Rigourous bounds for the Föpplvon Kármán theory of isotropically compressed plates. J. Nonlinear Sci. 10, 661-683 (2000)

5. Carstensen, C., Plecháč, P.: Numerical solution of the scalar double-well problem allowing microstructure. Math. Comp. 66, 997-1026 (1997)

6. Carstensen, C., Roubíček, T.: Numerical approximation of young measures in non-convex variational problems. Numer. Math. 84, 395-415 (2000)

7. Chipot, M., Collins, C.: Numerical approximations in variational problems with potential wells, SIAM J. Numer. Anal. 29, 1002-1019 (1992)

8. Chipot, M.: The appearance of microstructures in problems with incompatible wells and their numerical approach. Numer. Math. 83, 325-352 (1999)

9. Chipot, M., Collins, C., Kinderlehrer, D.: Numerical analysis of oscillations in multiple well problems. Numer. Math. 70, 259-282 (1995)

10. Chipot, M., Müller, S.: Sharp energy estimates for finite element approximations of non-convex problems in: Variations of domain and free-boundary problems in solid mechanics (Paris), pp. 317-325 (1997)

11. Ciarlet, P.G.: The Finite Element Method for Elliptic Problems. Amsterdam: NorthHolland, 1978

12. Matthias, G.K., Prohl, A.: A discontinuous finite element method for solving a multiwell problem. SIAM J. Numer. Anal. 37, 246-268 (1999) 
13. Kohn, R.V., Müller, S.: Branching of twins near an austenite/twinned martensite interface. Phil. Mag. 66A, 697-715 (1994)

14. Kohn, R.V., Müller, S.: Surface energy and microstructure in coherent phase transitions. Comm. Pure Appl. Math. 47, 405-435 (1994)

15. Kružik, M.: Numerical approach to double well problems. SIAM J. Numer. Anal. 35, 1833-1849 (1998)

16. Li, B.: Finite element analysis of a class of stress-free martensitic microstructures. Math. Comp., 2002. In press

17. Li, B., Luskin, M.: Nonconforming Finite element approximation of crystalline microstructure. Math. Comp. 67, 917-946 (1998)

18. Li, B., Luskin, M.: Theory and computation for the microstructure near the interface between twinned layers and a pure variant of martensite. Mat. Sci. Eng. A, 273-275, 237-240 (1999)

19. Luskin, M.: Approximation of a laminated microstructure for a rotationally invariant, double well energy density. Numer. Math. 75, 205-221 (1997)

20. Luskin, M.: On the computation of crystalline microstructure. Acta Numerica, 1996

21. Müller, S.: Variational models for microstructure and phase transitions. Springer. Lect. Notes Math. 1713, 85-210 (1999)

22. Pedregal, P.: On the numerical analysis of nonconvex variational problems. Num. Math. 74, 325-336 (1996)

23. Prohl, A.: An adaptive finite element method for solving a double well problem describing crystalline microstructure. M2AN 33, 781-796 (1999)

24. Shield, T.W.: Needles in Martensites. www.aem.umn.edu/people/faculty/shield/ needles 